\title{
An Experimental Investigation of Enhanced SM-OFDM Over Indoor Rician Multipath Channels
}

\author{
Ke Jiang, Zilong Liu, Senior Member, IEEE, Ping Yang, Senior Member, IEEE, Yue Xiao, Member, IEEE, and \\ Shaoqian Li, Fellow, IEEE,
}

\begin{abstract}
In this letter, we investigate enhanced spatial modulation (ESM) aided orthogonal frequency division multiplexing (OFDM) systems. ESM extends the conventional SM by introducing a series of signal constellations. Existing research shows that ESM displays performance superiority over SM in flat-fading channels. For frequency-selective fading channels, we design a practical implementation of an ESM\&SM aided OFDM hybrid system to characterize the bit error rate (BER) performance of ESM and conventional SM with an experimental wireless testbed. Form the experimental results, it is found that ESM achieves better BER performance than SM on frequency-selective channels, which are consistent with the Monte Carlo simulation results.
\end{abstract}

Index Terms-Bit error rate (BER), enhanced spatial modulation (ESM), multiple-input multiple-output orthogonal frequency division multiplexing (MIMO-OFDM), wireless testbed.

\section{INTRODUCTION}

$\mathbf{M}$ ULTIPLE-INPUT multiple-output orthogonal frequency division multiplexing (MIMO-OFDM) [1] is a key enabling technique for modern high-rate communications. However, due to the use of multiple-antenna and multiplecarrier, current MIMO-OFDM suffers from several problems, such as the inter-channel interference (ICI), the inter-antenna interference (IAI), the need for multiple radio frequency (RF) chains [2], and the high peak-to-average power ratio (PAPR) of the transmitted signals. These problems result in extra complexity or power burden to MIMO-OFDM system design.

Recently, spatial modulation-OFDM (SM-OFDM) has been proposed to address some of the above mentioned problems pertinent to MIMO-OFDM. The main idea of SM-OFDM is that at each channel use only one single transmit antenna (TA) activates its subcarrier for data transmission. The sparsity of the transmit vectors in frequency domain helps lower the PAPR, reduce the ICI, and allow a low-complexity receiver [2]-[4]. To date, some intensive studies have been conducted on SM-OFDM [5]-[7]. To be specific, [5] studied the average bit error rate (BER) performance of SM-OFDM with the socalled $N$-continuous (NC) precoder under perfect and imperfect channel estimations. [6] proposed a detection scheme for SM-OFDM which can effectively mitigate the effect of

K. Jiang, P. Yang, Y. Xiao and S. Li are with the National Key Laboratory of Science and Technology on Communications, University of Electronic Science and Technology of China 611731, Sichuan, China. (Email: yang.ping, xiaoyue, lsq@uestc.edu.cn).

Z. Liu was with the $5 \mathrm{G}$ Innovation Centre, Institute for Communication Systems, University of Surrey, UK. He is now with the School of Computer Science and Electronic Engineering, University of Essex, UK. (Email: zilongliu.sg@gmail.com)

The corresponding author is Ping Yang.

This work is supported by the National Key R\&D Program of China under Grant 2017YFE0121500, and the National Science Foundation of China under Grant number 61876033. The work of Z. Liu was also supported in part by National Natural Science Foundation of China (Grant No. 61750110527), Research Fund for International Young Scientists. the carrier frequency offset (CFO) at a slight complexity increase. Gong et al. [7] applied SM-OFDM to provide robust communications over high speed train (HST) systems.

Since only one tone is active to transmit data symbols at each channel use, the transmission rate of SM-MIMO may not compete with that of V-BLAST MIMO, in which all the TAs are active to transmit data symbols simultaneously. This implies that a larger number of TAs are required for SMMIMO to achieve the same transmission rate as that of $\mathrm{V}$ BLAST MIMO; when a small number of TAs are equipped, it may be hard for SM-MIMO to achieve a high transmission rate.

Motivated by the throughput loss of SM-MIMO, a promising variant of SM-MIMO, named enhanced SM-MIMO (ESMMIMO), has been proposed [8]. The main feature of ESMMIMO is that the number of the active TAs can be one or two. When only one TA is active, a data symbol from the primary signal constellation is transmitted, while when two TAs are active, two data symbols from the secondary constellation are transmitted simultaneously in parallel. In ESM-MIMO, the transmission with two active TAs leads to a rate increase, while the properly designed primary and secondary constellations bring an improvement of BER performance.

To the best of our knowledge, none of the existing studies on ESM have been validated on testbed implementation, except for conventional SM. [9] may be the first time to analyze the performance of SM using practical channel measurement. [10], [11] investigated the BER performance of SM by channel measurements in an indoor propagation scenario, and validated the performance superiority of SM over other conventional MIMO schemes, such as spatial multiplexing (SMX), spacetime block code (STBC) in both line-of-sight (LOS) and none line-of-sight (NLOS) scenarios. Moreover, most of the current studies on ESM are focused on the scenarios of flat-fading channels, and less has been understood on the performance of ESM in frequency-selective fading channels.

Against the above background, we summarize the main contributions of this work as follows.

1) We apply ESM to OFDM transmission, make comparisons of BER performance between the ESM-OFDM and SM-OFDM over Rician multipath channels and derive the related performance metrics with differen$\mathrm{t}$ configurations. From the performance metrics and Monte Carlo simulations, it is found that ESM-OFDM outperforms SM-OFDM in frequency-selective channels.

2) we design a hardware prototype based on National Instruments Universal Software Radio PeripheralReconfigurable Input / Output (NI USRP-RIO) platform to validate the BER performance of ESM-OFDM and SM-OFDM. Our experimental results verify the perfor- 

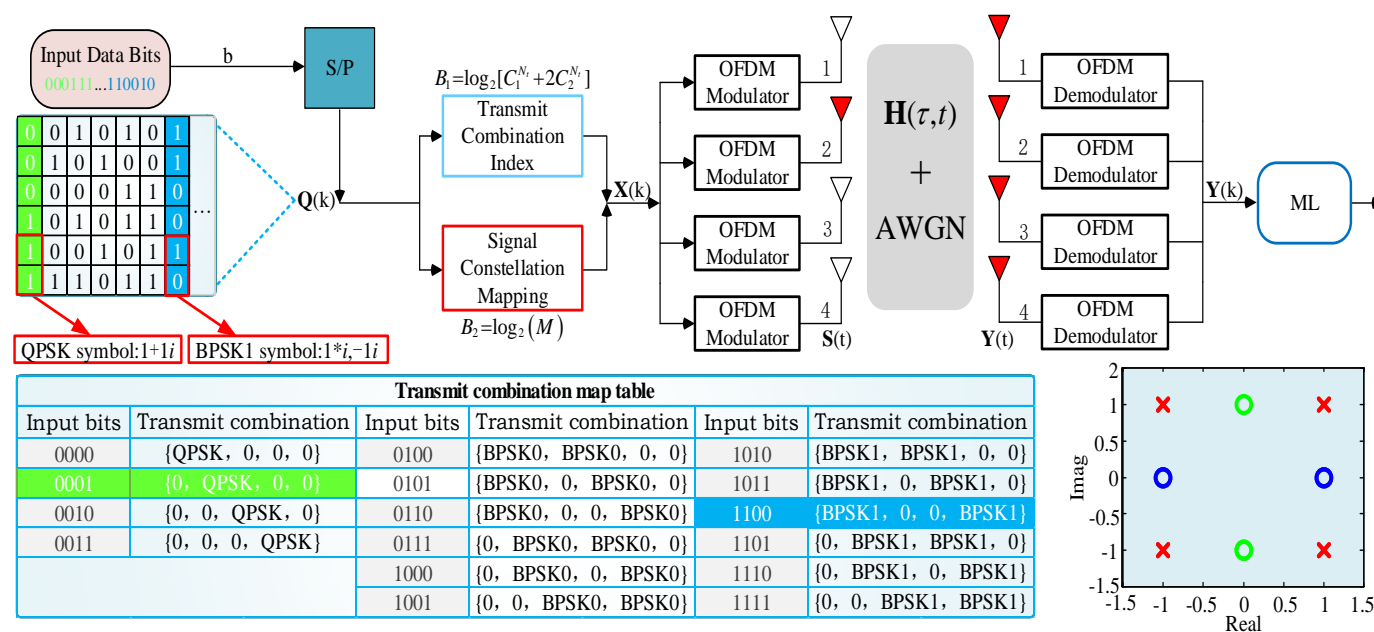

Fig. 1. An illustration of ESM-OFDM system. All available transmit combinations are listed in the table.

mance superiority of ESM-OFDM over SM-OFDM in practical indoor multipath scenarios.

Notation: $(\cdot)^{T},(\cdot)^{H},\|\cdot\|_{\mathrm{F}}$, and $|\cdot|$ denote transpose, Hermitian transpose, Frobenius norm and determinant, respectively. $\mathcal{C N}(0,1)$ denotes the complex Gaussian distribution with zero mean and unit variance. $C_{n}^{k}$ is the binomial coefficient. The argument of $z$ is $\arg (z)$ and $z^{*}$ is the complex conjugate of $z$.

\section{System Model AND Testbed Setup}

\section{A. System Model}

As shown in Fig. 1, we consider an ESM-OFDM system with $N_{t}=4$ TAs and $N_{r}=4$ receive antennas (RAs), in which the $M$-ary amplitude and phase modulation ( $M$-PAM) is the primary constellation. The input data bitstream is firstly converted into a $B \times N$ matrix $\mathbf{Q}(k)$, where

$$
B=B_{1}+B_{2}=\log _{2}\left[C_{1}^{N_{t}}+2 C_{2}^{N_{t}}\right]+\log _{2}(M)
$$

is the number of bits of each ESM unit, and $N$ is the number of OFDM subcarriers. Then, each unit is split into transmit combination index part and data symbol part. The former part decides the combination of active TAs and signal constellation. If the former indicates only one active TA, the latter (all $B_{2}$ bits) is mapped to a primary constellation point; or the latter is further split into two parts ( $\frac{B_{2}}{2}$ bits of each), mapped to two dada symbols from the same secondary $\frac{M}{2}$-PAM constellation and transmitted simultaneously in parallel. Based on above principle, there are two types of ESM transmit vectors, i.e.

$$
\mathbf{x}_{1}=s_{m}^{q} \mathbf{e}_{q}=\left[0, \ldots, s_{\uparrow q \text { th }}^{q}, \ldots, 0\right]^{T},
$$

and

$$
\mathbf{x}_{2}=s_{n_{1}}^{i} \mathbf{e}_{i}+s_{n_{2}}^{j} \mathbf{e}_{j}=\left[0, \ldots, s_{n_{1}}^{i}, \ldots, \underset{\uparrow i \text { th }}{\substack{n_{n_{2}} \\ j}}, \ldots, 0\right]^{T}(i \neq j),
$$

where $s_{m}^{q}(1 \leq m \leq M)$ is a point from the primary constellation signal set $\mathbb{S}_{1}$, while $s_{n_{1}}^{i}, s_{n_{2}}^{j}\left(1 \leq n_{1}, n_{2} \leq \frac{M}{2}\right)$ are from the same $\frac{M}{2}$-ary secondary signal set $\mathbb{S}_{2}$. Moreover, $\mathbf{e}_{q}, \mathbf{e}_{i}, \mathbf{e}_{j}$ $\left(1 \leq q, i, j \leq N_{t}\right)$ are the $q$ th, $i$ th, $j$ th column vectors of an $N_{t} \times N_{t}$ identity matrix, respectively.

For further clarification, Fig. 1 shows examples of $4 \times 4$ ESM in which QPSK is adopted as the primary signal constellation. For the first column of $\mathbf{Q}(k), \mathbf{Q}_{1}(k)=[0,0,0,1,1,1]^{T}$, the corresponding output vector is $\mathbf{x}_{1}=[0,1+1 i, 0,1]^{T}$. Let us consider the case of two active TAs. By taking the seventh column of $\mathbf{Q}(k), \mathbf{Q}_{7}(k)=[1,1,0,0,1,0]^{T}$ as an example, the corresponding output vector is $\mathbf{x}_{7}=[1 i, 0,0,-1 i]^{T}$.
After ESM mapping, each row of symbol matrix $\mathbf{X}(k)=$ $\left[\mathbf{x}_{1}(k), \mathbf{x}_{2}(k), \ldots, \mathbf{x}_{N}(k)\right]$ is sent into an OFDM modulator, and the output is a row of the transmit signal matrix $\mathbf{S}(t)$. The transmit signal $\mathbf{S}(t)$ goes through the multipath channel $\mathbf{H}(\tau, t)=\left[\hat{\mathbf{h}}_{n_{r}, n_{t}}(\tau, t)\right][3]$ superimposed by the additive white Gaussian noise (AWGN), where

$$
\hat{\mathbf{h}}_{n_{r}, n_{t}}(\tau, t)=\left[h_{n_{r}, n_{t}}^{(1)}\left(\tau_{1}, t\right), h_{n_{r}, n_{t}}^{(2)}\left(\tau_{2}, t\right), \ldots, h_{n_{r}, n_{t}}^{(l)}\left(\tau_{l}, t\right)\right]^{T}
$$

is a $l \times 1$ channel impulse response vector, $\tau_{\phi}=r_{\phi} / c(\phi=$ $\left.1,2, \ldots, l, c=3 \times 10^{8} \mathrm{~m} / \mathrm{s}\right)$ is the propagation delay of the $\phi$ th channel path with a path length of $r_{\phi} . l$ is the number of multipath components between the $n_{t}$ th TA and $n_{r}$ th RA $\left(1 \leq n_{r} \leq N_{r}, 1 \leq n_{t} \leq N_{t}\right)$. Due to the LOS component, the channel impulse response between each pair of RA and TA is the sum of a fix component and a random multipath component,

$$
\hat{\mathbf{h}}_{n_{r}, n_{t}}(\tau, t)=\sqrt{\frac{K}{K+1}} \mathbf{h}_{n_{r}, n_{t}}^{\operatorname{LOS}}+\sqrt{\frac{1}{K+1}} \mathbf{h}_{n_{r}, n_{t}}(\tau, t),
$$

where $\mathbf{h}_{n_{r}, n_{t}}^{\mathrm{LOS}}=[1,0, \ldots, 0]^{T}$ is a $1 \times l$ vector, whose first entry is one and others are zero, $\mathbf{h}_{n_{r}, n_{t}}(\tau, t)$ is a circularly symmetric complex Gaussian random variable of $\mathcal{C N}(0,1)$, and $K$ is the Rician factor. At the receiver, the received signal is

$$
\mathbf{Y}(t)=\mathbf{H}(\tau, t) \otimes \mathbf{S}(t)+\mathbf{Z}(t),
$$

where $\otimes$ denotes time domain convolution and $\mathbf{Z}(t)$ denotes the AWGN. $N_{r}$ rows of $\mathbf{Y}(t)$ are demodulated by $N_{r}$ OFD$\mathrm{M}$ demodulators, where the zero-forcing frequency-domain equalizer is used, and the output is an $N_{r} \times N$ matrix $\mathbf{Y}(k)$. When the number of transmit antennas is relatively small and the discrete time-invariant frequency response channel matrix $\mathbf{H}(k)$ is perfectly known at the receiver, a maximum likelihood (ML) detector is deployed. For the $\rho$ th column of $\mathbf{Y}(k)$,

$$
\mathbf{y}_{\rho}(k)=\mathbf{H}(k) \mathbf{x}_{\rho}(k)+\mathbf{z}(k),
$$

the detection can be modeled as the following two cases:

$$
\begin{aligned}
{[\hat{q}, \hat{m}] } & =\arg \min _{q, m}\left\|\mathbf{y}_{\rho}(k)-\mathbf{H}(k) \mathbf{x}_{\rho}(k)\right\|_{\mathrm{F}}^{2}, \\
{\left[\hat{i}, \hat{j}, \hat{n}_{1}, \hat{n}_{2}\right] } & =\arg \min _{i, j, n_{1}, n_{2}}\left\|\mathbf{y}_{\rho}(k)-\mathbf{H}(k) \mathbf{x}_{\rho}(k)\right\|_{\mathrm{F}}^{2} .
\end{aligned}
$$

Because the number of active TAs can be 1 or 2 in ES$\mathrm{M}$ and the transmitted constellation symbols are from the primary $\left(\mathbb{S}_{1}\right)$ or secondary $\left(\mathbb{S}_{2}\right)$ signal constellation set, the ML detection selects the optimal solution $[\hat{q}, \hat{m}]$ or $\left[\hat{i}, \hat{j}, \hat{n}_{1}, \hat{n}_{2}\right]$ corresponding to the minimum of (8) and (9). 

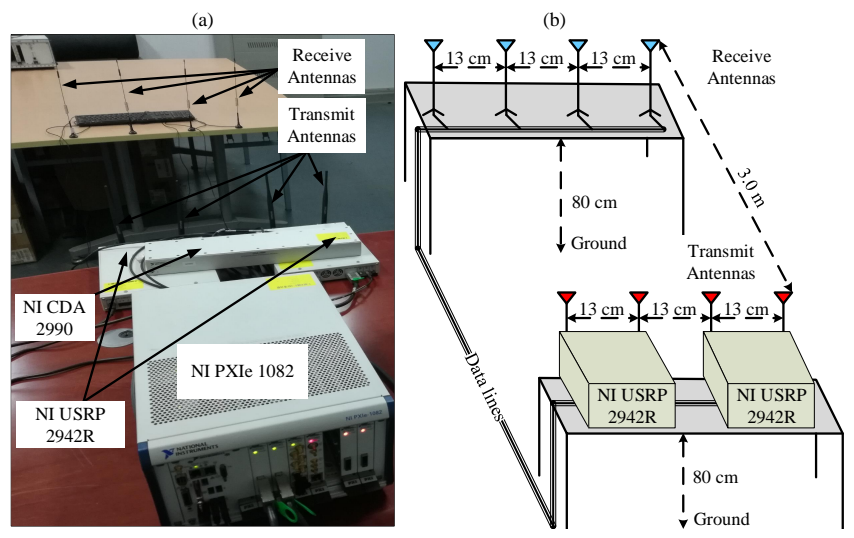

Fig. 2. (a) Experimental setup in the laboratory; (b) Physical experimental layout. The RAs set and the TAs set are set $3.0 \mathrm{~m}$ apart from each other with a direct LOS. Each set of omnidirectional antennas is $80 \mathrm{~cm}$ from the ground, and there is a $13 \mathrm{~cm}$ distance between each pair of neighbouring antennas, which is sufficient to guarantee very low spatial correlation.

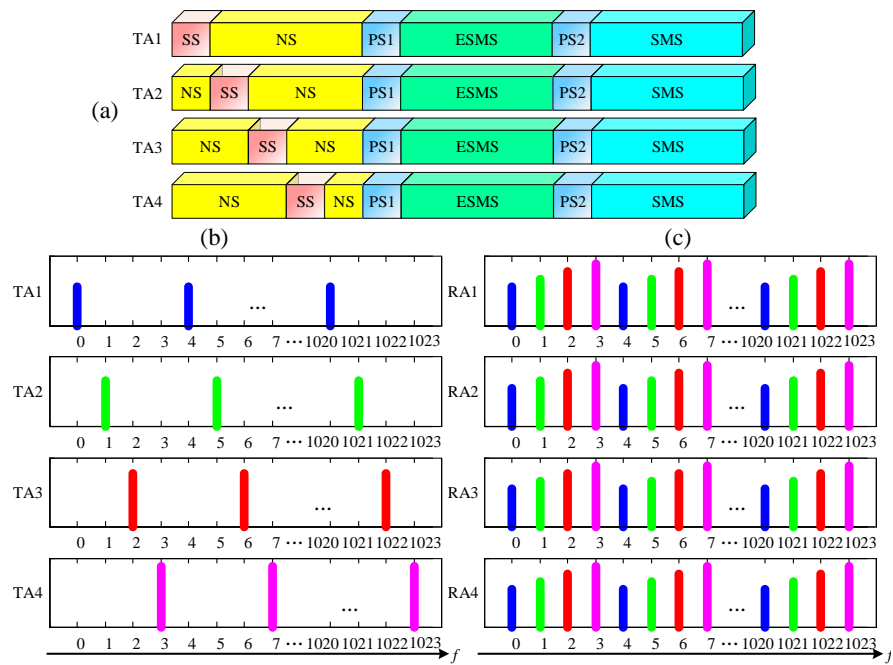

Fig. 3. (a) Data frame structure in each TA. Each frame consists of 15 OFDM symbols; (b) PS structure of the transmitted signals. The PS is orthogonal to each other in frequency domain; (c) PS structure for received data frames. Mixed PS are used to estimate the 4 corresponding channels for each RA.

\section{B. Testbed Setup and Measurement Campaign}

Our testbed setup and physical experimental layout for the $4 \times 4$ practical implementation system are illustrated in Fig. 2 (b). $2.2368 \times 10^{6}$ bits are transmitted repeatedly to obtain the experimental results. Data bits are arranged in 92 frames with 24576 bits each, then mapped to a $4 \times 1024$ data symbol matrix. As shown in Fig. 3 (a), each data frame consists of synchronization sequence (SS) of 1024 symbols, whose last 512 symbols are copies of the first 512 symbols, noise sequence (NS) of 5120 zeros, ESM sequence (ESMS) of 4096 symbols, two pilot sequences (PS) of 256 pilot symbols and 768 zeros, and SM sequence (SMS) of 4096 symbols. After IFFT, each OFDM symbol is added a cyclic prefix of 40 symbols, and before pulse shaping, each data frame is added another 120 zeros.

1) Testbed Hardware: As shown in Fig. 2, main part of the tested system is a NI PXIe 1082. Two NI USRP 2924R connected to the NI PXIe 1082 are employed as transmitter and receiver. A NI CDA-2990 is used to synchronize these two USRP-RIO devices.
2) Testbed Software: LabVIEW 2014 64bit is used to facilitate the transmitter link and the receiver link. The transmitter consists of framing module, ESM/SM module, PS and SS insert module, IFFT module, pulse shaping module. The receiver consists of frame synchronization module, frequency offset correction module, FFT module, channel estimation module, SNR calculation module, ML detection module, BER calculation module. Limited by the length of the paper, we can only describe the details of some of the key modules of the testbed systems links in the following part.

- Coarse Synchronization: In order to acquire the coarse starting point of the transmitted data, a special sequence is firstly transmitted repeatedly 16 times to inform the receiver that the transmission is going to start. BER of the special sequence is calculated in the receiver using the recovered and local special sequence, and the transmitter will not transmit data sequences until the calculated BER for special sequence is lower than a specific threshold. From plenty of experiments, we found that it is more proper to set the threshold to 0.2 .

- Frame Synchronization: Inspired by the algorithm proposed in [13], here a cross-correlation based time synchronization algorithm is used, and we extend this algorithm to MIMO-OFDM systems. A Zadoff-Chu (ZC) sequence of 1024 symbols is added to the start of each data frame from each TA, which is defined by [14]

$$
d_{u}(n)=e^{-j \frac{\pi u n(n+1)}{N_{Z C}}}, n=0,1, \ldots, N_{Z C}-1,
$$

where the ZC root sequence index $u$ is $25, N_{Z C}=512$. The SS corresponding to each TA is orthogonal to each other, which is achieved by cyclic shift of $d_{u}(n)$. Assume the SS for the 1st TA is $d_{u}^{(1)}(n)$, then the SS corresponding to the $q$ th $\left(1 \leq q \leq N_{t}\right)$ TA is

$$
d_{u}^{(q)}(n)=d_{u}^{(1)}(n) e^{j \frac{2 \pi q\left\lfloor N_{Z C} / N_{t}\right\rfloor}{i N_{Z C}}}, i=1,2, \ldots, N_{Z C},
$$

Step I: The receiver firstly samples $M_{s}=32160$ data symbols, i.e. the length of two data frames (15 OFDM symbols with $\mathrm{CP}$ and 120 zeros), aiming to extract at least one complete data frame. Then sliding correlation is conducted between the local SS and the sampled signal to achieve cross-correlation to obtain the timing point of current sampled signal frames. The cross-correlation can be expressed as:

$$
\operatorname{Cor}(\theta)=\left|\sum_{k=0}^{N} r(\theta+k) c^{*}(k)\right|^{2}, \theta=0,1, \ldots, M_{s}-N-1,
$$

where $c(k)$ is the known local SS of length $N=1024$, $r(k)$ is the sampled received signal and the timing point $\hat{\theta}$ is given by the peak of $\operatorname{Cor}(\theta)$. Theoretically, there are two peak points in the cross-correlation output and the index of the first peak indicates the timing point of the sampled data frame.

Step II: Moreover, for the signal received by each RA, the above procedures are similar. Due to the special structure shown in Fig. 3 (a), we can check the accuracy by calculating the different values of the timing points and compare these different values with theoretical values. Theoretically, the different value between the timing points of each pair of signals received by each pair of adjacent RAs is 1064 . Timing points corresponding to 
the 4 TAs are $\hat{\theta}_{1}=5, \hat{\theta}_{2}=1069, \hat{\theta}_{3}=2133, \hat{\theta}_{4}=3197$ in our testbed system. If the different values of some data frame do not consistent with the theoretical values, this data frame do not need to go through the frame synchronization repeatedly until reach consistentence.

- CFO Estimation: With perfect frame synchronization, a carrier frequency offset (CFO) of $\varepsilon$ causes a phase rotation of $2 \pi i \varepsilon / N$ in received signals. A training sequence based CFO estimation algorithm is exploited and the SS is used as the training sequence. The CFO estimation is achieved by the auto-correlation of the SS of each data frame. After frame synchronization, the SS of the extracted frame is abstracted and cut the CP away. Then the receiver makes the CFO estimation utilizing the repetitive pattern of SS as follows [13]:

$$
\hat{\varepsilon}=\frac{N}{2 \pi N_{Z C}} \arg \sum_{k=0}^{\frac{N}{2}-1}\left\{r(\hat{\theta}+k) r^{*}\left(\hat{\theta}+k+\frac{N}{2}\right)\right\},
$$

Next the CFO of received data frame is corrected by multiplying $e^{-\mathrm{j} \frac{2 \pi i}{N} \hat{\varepsilon}}, i=1,2, \ldots, N$.

- Channel Estimation: Here the linear minimum mean square error (LMMSE) channel estimation algorithm [15] is used. For each pair of TA and RA, we have

$$
\mathbf{Y}=\mathbf{X H}+\mathbf{Z}
$$

where $\mathbf{X}=\operatorname{diag}\left(X_{1}, X_{2}, \ldots, X_{N-1}\right)$ is the PS, $X_{k}$ is the pilot symbol on the $k$ th subcarrier $E\left\{X_{k}\right\}=0, D\left\{X_{k}\right\}=$ $\sigma_{x}^{2}, \mathbf{H}=\left[H_{1}, H_{2}, \ldots, H_{N-1}\right]^{T}$ is the channel vector, $\mathbf{Z}=$ $\left[Z_{1}, Z_{2}, \ldots, Z_{N-1}\right]^{T}$ is the noise vector with mean zero and variance $\sigma_{z}^{2}$, so the estimated channel matrix is

$$
\hat{\mathbf{H}}_{L M M S E}=\mathbf{R}_{\mathbf{H H}}\left(\mathbf{R}_{\mathbf{H H}}+\alpha \beta \mathbf{I}_{N}\right)^{-1} \mathbf{X}^{-1} \mathbf{Y},
$$

where $\alpha=\sigma_{z}^{2} / E\left\{\left|1 / X_{k}\right|^{2}\right\}, \beta=E\left\{\left|X_{k}\right|^{2}\right\} E\left\{\left|1 / X_{k}\right|^{2}\right\}$, $\tau_{r m s}=50$ ns, $\mathbf{R}_{\mathbf{H H}}=E\left\{\mathbf{H H}^{\mathrm{H}}\right\}=\left[r_{m, n}\right]$,

$$
r_{m, n}=\frac{1-e^{-L\left(\tau_{r m s}^{-1}+2 \pi j(m-n) / N\right)}}{\left(1-e^{-L \tau_{r m s}^{-1}}\right)\left(1+2 \pi \tau_{r m s} j(m-n) / N\right)} \text {. }
$$

As shown in Fig. 3 (b), each PS is extended to a sequence of 1024 symbols by inserting zeros and are added to data frame as shwon in 3 (a). And the sparse structure of the PS of transmitted signals makes it independent for the channel estimation of each pair of TA and RA. First of all, PS symbols at special subcarriers are extracted and used for estimating the corresponding 256 channel gains in special positions using LMMSE. Then the 256 estimated channel gains are interpolated linearly in frequency domain to obtain the other 768 channel gains for other subcarriers. Finally, the two complete channel estimation for the 8th and 13th OFDM symbols are linearly interpolated between each two corresponding channel gains which are corresponding to the same subcarrier to obtain the channel gains for other OFDM symbols.

- ML Detection: According to (8) or (9), the estimated channels are used for ML detection and the transmitted vectors are detected one by one. The ML search range is all the available transmit vectors and it evolves the detection of the index of active TAs and the transmitted symbols. Then the index of TAs and symbols are converted into bits and are connected in serial.
- SNR Calculation: In our testbed system, SNR is an output of the receiver and related system links are required for its calculation. Note that the noise in Matlab simulation is modelled as AWGN, while in the practical system, except for AWGN, there are some other types of noise, like the thermal noise. The SNR is defined as

$$
S N R(d B)=10 \log _{10} \frac{E\left\{\left\|\mathbf{H}(k) \mathbf{x}_{\rho}(k)\right\|_{\mathrm{F}}^{2}\right\}}{\sigma_{z}^{2}},
$$

where

$$
\begin{gathered}
E\left\{\left\|\mathbf{H}(k) \mathbf{x}_{\rho}(k)\right\|_{\mathrm{F}}^{2}\right\}= \\
\sum_{i=1}^{4096}\left(\left\|\mathbf{y}_{\rho, i}(k)\right\|_{\mathrm{F}}^{2}-\left\|\mathbf{z}_{i}\right\|_{\mathrm{F}}^{2}-2\left[\mathbf{y}_{\rho, i}(k)\right]^{H} \mathbf{z}_{i}\right), \\
\sigma_{z}^{2}=\sum_{i=1}^{1024}\left\|\mathbf{z}_{i}\right\|_{\mathrm{F}}^{2}-\left[\sum_{i=1}^{1024}\left\|\mathbf{z}_{i}\right\|_{\mathrm{F}}\right]^{2},
\end{gathered}
$$

We change the value of (18) by tuning the power of transmit vectors. Assume that OFDM symbols in one frame superimposed by the same noise, the NS in each data frame is used to measure the noise. And the power of the noise is calculated according to (19).

\section{Performance Metric and Complexity Comparison Analysis}

At high SNR regions, assuming over uncorrelated Rician channel, the asymptotic BER performance is determined by the worst pairwise error probability (PEP), which corresponds to the minimum of the squared Euclidean distance between each two transmit vectors $\mathbf{x}$ and $\mathrm{x}^{\prime}[16]$ :

$$
d_{\min }^{2}=\min _{\mathbf{x} \neq \mathbf{x}^{\prime}}\left\|\mathbf{x}-\mathbf{x}^{\prime}\right\|_{\mathrm{F}}^{2}
$$

Assume $E\left\{\mathbf{x}^{H} \mathbf{x}\right\}=1$, the $d_{\min }^{2}$ for ESM-OFDM that exploits QPSK or 16QAM as primary constellation, and the $d_{\text {min }}^{2}$ for SM-OFDM that exploits 16QAM or 64QAM are reported in TABLE I. Note that a larger minimum of the squared Euclidean distance between transmit vectors gives rise to a better BER performance. Due to the same OFDM modulation and demodulation for ESM-OFDM and SM-OFDM, we only consider the detection complexity for each subcarrier in complexity analysis part, and the complexity is defined as

TABLE I

$d_{\min }^{2}$ OF ESM VECTORS AND SM VECTORS WITH 4 TAS

\begin{tabular}{|c|c||c|c|}
\hline Scheme & $6 \mathrm{~b} / \mathrm{s}$ & Scheme & $8 \mathrm{~b} / \mathrm{s}$ \\
\hline SM-OFDM-16QAM & 0.4 & SM-OFDM-64QAM & 0.095 \\
\hline ESM-OFDM-QPSK & 1 & ESM-OFDM-16QAM & 0.36 \\
\hline
\end{tabular}

TABLE II

DETECTION COMPLEXITY

\begin{tabular}{|c|c||c|c|}
\hline Scheme & $6 \mathrm{~b} / \mathrm{s}$ & Scheme & $8 \mathrm{~b} / \mathrm{s}$ \\
\hline SM-OFDM-16QAM & 128 & SM-OFDM-64QAM & 512 \\
\hline ESM-OFDM-QPSK & 96 & ESM-OFDM-16QAM & 352 \\
\hline Reduction Rate & $25 \%$ & Reduction Rate & $31.3 \%$ \\
\hline
\end{tabular}

the number of complex multiplications needed for each ML detection [8]. According to Eq. (2)(3)(7), complex multiplications involved in

$z_{l}=y_{l}-h_{l q} s_{m}^{q}, z_{l}=y_{l}-h_{l i} s_{n_{1}}^{i}-h_{l j} s_{n_{2}}^{j}\left(1 \leq l \leq N_{t}\right)$,

for vectors $\mathbf{x}_{1}, \mathbf{x}_{2}$, and in

$$
\|\mathbf{z}(k)\|_{\mathrm{F}}^{2}=\left\|\mathbf{y}_{\rho}(k)-\mathbf{H}(k) \mathbf{x}_{\rho}(k)\right\|_{\mathrm{F}}^{2} \text {. }
$$

Detection complexity at different given transmission rate are reported in TABLE II. As shown in TABLE II, ESM greatly 
helps reduce the numbers of complex multiplications needed for detection. The complexity reduction of ESM-OFDM with respect to SM-OFDM is obvious and the reduction rate are $25 \%, 31.3 \%$ for $6 \mathrm{bits} / \mathrm{symbol}(\mathrm{b} / \mathrm{s}), 8 \mathrm{~b} / \mathrm{s}$, respectively.

\section{Results And AnAlysis}

In this section, we present the Monte Carlo simulation results and the experimental results to evaluate the BER performance of ESM-OFDM and SM-OFDM with the same antenna configuration of $N_{t}=N_{r}=4$ on indoor Rician multipath fading channels in Fig. 4. Moreover, for fair comparison, we add GSM-OFDM with fixed number of active TA into the comparison. The carrier frequency $f=3.5 \mathrm{GHz}$, the number of OFDM subcarrier $N=1024$, subcarrier spacing $\triangle f=15$ $\mathrm{KHz}$, sampling frequency $f_{s}=15.36 \mathrm{MHz}$ and the length of $\mathrm{CP}$ is 40 . The path delay $\tau$ vector used is modified form [12]:

$$
\begin{aligned}
& \tau=\left[\left[\begin{array}{lllllllllllll}
0 & 10 & 20 & 30 & 40 & 50 & 60 & 70 & 80 & 90 & 100 & 120 & \ldots
\end{array}\right.\right. \\
& 140160200240280320]] \times 10^{-9}(s)
\end{aligned}
$$

and $K=33$ is modified from [11] according to comparison of the Monte Carlo simulation results and the experimental results. Fig. 4 (a) shows the comparison of BER performance for ESM-OFDM, SM-OFDM and GSM-OFDM at the same transmission rate $6 \mathrm{~b} / \mathrm{s}$. And the comparison of experimental results and Monte Carlo simulation results are also depicted. Fig. 4 (b) compares the results of ESM-OFDM, SM-OFDM and GSM-OFDM at the transmission rate of $8 \mathrm{~b} / \mathrm{s}$. It is seen that ESM-OFDM outperforms the SM-OFDM and GSMOFDM at high SNR regions. It can also be found that when higher order signal constellations are adopted, there is a performance loss due to the minimum squared Euclidean distance between transmit vectors gets smaller. One can see that the experimental results approximate the simulation results, especially at high SNR regions. Performance gap between the experimental results curves and the simulation curves can be attributed to hardware imperfections, channel estimation error, SNR calculation method, and other interfering signals from the experimental environment. And the multipath channel parameters in the Monte Carlo simulation, such as the value of the $K$ factor, number of multipath, path delay vectors, average path gains vectors may not consistent with the real channel condition exactly. Based on our results and the results in [10], ESM-OFDM is a promising candidate for future indoor MIMO communications, compared to SM-OFDM and VBLAST-OFDM.

\section{CONCLUSION}

In this paper, we investigate the ESM aided OFDM transmission scheme, and compare it with SM-OFDM and GSMOFDM. From the calculations of minimum squared Euclidean distance between transmit vectors, the ESM-OFDM is proven to have better BER performance, compared to SM-OFDM and GSM-OFDM. And the detection reduction of ESM-OFDM respect to SM-OFDM is obvious. Under given transmission rate, both of the Monte Carlo simulation results and experimental results show that the ESM-OFDM outperforms the SM-OFDM and GSM-OFDM over indoor Rician multipath fading channels at high SNR regions.
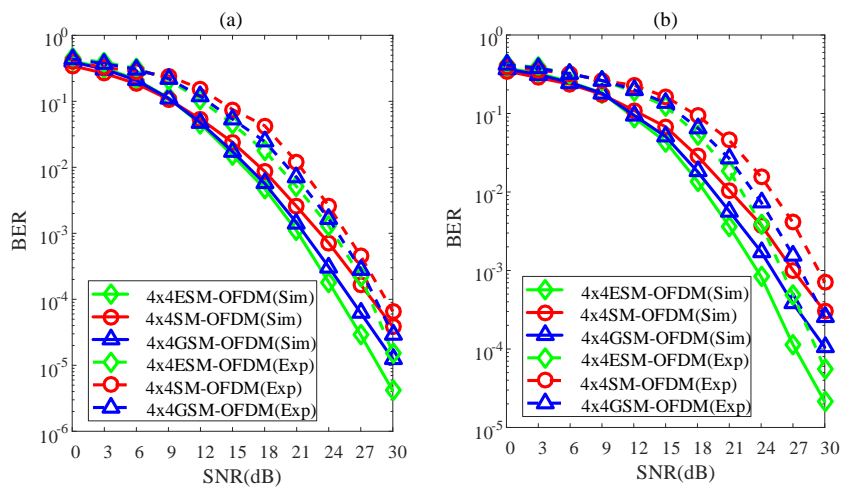

Fig. 4. The dotted lines represent the experimental (Exp) results and solid lines represent the Monte Carlo simulation (Sim) results. $N_{t}=N_{r}=4$. (a) BER comparison of the ESM-OFDM, SM-OFDM and GSM-OFDM at the transmission rate of $6 \mathrm{~b} / \mathrm{s}$. (b) BER comparison of the ESM-OFDM, SMOFDM and GSM-OFDM at the transmission rate of $8 \mathrm{~b} / \mathrm{s}$.

\section{REFERENCES}

[1] Y. Li, J. Winters, and N. Sollenberger, "MIMO-OFDM for wireless communications: Signal detection with enhanced channel estimation,' IEEE Trans. Commun., vol. 50, no. 9, pp. 1471-1477, Sep. 2002.

[2] P. Yang, Y. Xiao, M. Xiao, et al, "Adaptive spatial modulation MIMO based on machine learning, " IEEE J. Select. Areas Commun., vol. 37, no. 9, pp. 2117-2131, Sep. 2019.

[3] P. Yang, Y. Xiao, Y. L. Guan, et al,"Multidomain index modulation for vehicular and railway Communications: A survey of novel Techniques," IEEE Veh. Technol. Mag., vol.13, no. 3, pp. 124-134, Sep. 2018.

[4] P. Yang, Y. Xiao, M. Xiao and S. Li, "6G wireless communications: Vision and potential techniques," IEEE Network, vol. 33, no. 4, pp. 70-75, Jul. 2019.

[5] Y. Xiao, F. Yu, X. Lei, et al, "Performance analysis for the $N$ continuous precoded SM-OFDM system," IEEE Trans. Veh. Technol., vol. 67, no. 1, pp. 483-493, Jan. 2018.

[6] Y. Zhao, Y. Xiao, B. Dong, et al, "Efficient detection of spatial modulation OFDM systems with multiple carrier frequency offsets," IEEE Commun. Lett., vol. 21, no. 2, pp. 426-429, Feb. 2017.

[7] B. Gong, L. Gui, Q. Qin and X. Ren, "Compressive sensing-based detector design for SM-OFDM massive MIMO high speed train systems,' IEEE Trans. Broadcast., vol. 63, no. 4, pp. 714-726, Dec. 2017.

[8] C.-C. Cheng, H. Sari, S. Sezginer, and Y. T. Su, "Enhanced spatial modulation with multiple signal constellations," IEEE Trans. Commun. vol. 63, no. 6, pp. 2237-2248, Jun. 2015.

[9] A. Younis, W. Thompson, M. D. Renzo, et al, "Performance of spatial modulation using measured real-world channels," in Proc. 78th IEEE VTC, Las Vegas, NV, USA, 2013, pp. 1-5

[10] J. Zhang, Y. Wang, L. Ding, and N. Zhang, "Bit error probability of spatial modulation over measured indoor channels," IEEE Trans. Wireless Commun., vol. 13, no. 3, pp. 1380-1387, Mar. 2014.

[11] N. Serafimovski, A. Younis, R. Mesleh, et al, "Practical implementation of spatial modulation," IEEE Trans. Veh. Technol., vol. 62, no. 9, pp. 4511-4523, Nov. 2013.

[12] J. Medbo and P. Schramm, "Channel models for HIPERLAN/2 in different indoor scenarios," Etsi Doceri085b Eur. telecom. standards Inst, 1998.

[13] Y. S. Cho, J. Kim, W. Y. Yang, et al, MIMO-OFDM Wireless Communications with MATLAB, 2 Clementi Loop, Singapore: John Wiley \& Sons (Asia) Pte Ltd, 2010.

[14] TS 38.211 (V15.5.0) "NR: Physical Channels and Modulation", Mar. 2019.

[15] V. Savaux and Y. Louet, "LMMSE channel estimation in OFDM context: A review," IET Signal Process., vol. 11, no. 2, pp. 123-134, Api. 2017.

[16] A. Hedayat, H. Shah, and A. Nosratinia, "Analysis of space-time coding in correlated fading channels," IEEE Trans. Wireless Commun., vol. 4, no. 6, pp. 2882-2891, Nov. 2005 